\title{
Avaliação da efetividade do método de Tanaka-Johnston para predição do diâmetro mésio-distal de caninos e pré-molares não-irrompidos ${ }^{\dagger}$
}

\section{Evaluation of the effectiveness of the Tanaka-Johnston method for prediction of the mesiodistal diameter of unerupted canines and premolars}

\author{
Viviane Maria Teixeira MARCHIONNI* \\ Maria Catarina de Araújo SILVA* \\ Telma Martins de ARAÚJO** \\ Silvia Regina de Almeida REIS***
}

\begin{abstract}
MARCHIONNI, V. M. T.; SILVA, M. C. de A.; ARAÚJO, T. M. de; REIS, S. R. de A. Avaliação da efetividade do método de Tanaka-Johnston para predição do diâmetro mésio-distal de caninos e pré-molares não-irrompidos. Pesqui Odontol Bras, v. 15, n. 1, p. 35-40, jan./mar. 2001.

No presente estudo, os autores avaliaram o método de predição de Tanaka-Johnston com o objetivo de verificar sua efetividade para os lados direito e esquerdo, para ambos os sexos e para as raças branca, mulato claro, mulato médio, mulato escuro e negra, na cidade de Salvador - Bahia. Com o auxílio de um paquímetro digital, mediu-se o diâmetro mésio-distal dos incisivos inferiores permanentes, caninos e pré-molares, já irrompidos na cavidade bucal, de 98 indivíduos (45 do sexo masculino e 53 do sexo feminino), com idade variando entre 13 e 29 anos. Através de testes estatísticos, compararam-se os resultados obtidos a partir da aplicação das fórmulas de Tanaka-Johnston com os valores reais. Após análise dos resultados, observou-se que a correlação entre os valores estimados pela técnica de Tanaka-Johnston e os valores reais foram maiores para o sexo feminino que para o sexo masculino. Em relação à raça, os coeficientes de correlação foram satisfatórios para todas, sendo maior no arco dentário superior para a raça mulato escuro $(0,67)$ e no arco dentário inferior para a raça mulato claro $(0,74)$. Considerando-se os lados, os achados revelaram um maior coeficiente de correlação para o arco dentário inferior do lado esquerdo $(0,61)$. Pôde-se concluir, neste trabalho, que o método de Tanaka-Johnston, apesar de ter sido preconizado a partir de uma amostra de descendentes europeus, é indicado para predição do diâmetro mésio-distal de caninos e pré-molares não-irrompidos para diferentes raças, sexos e lados dos arcos dentários na amostra estudada.
\end{abstract}

UNITERMOS: Dentição mista; Ortodontia interceptora; Dente não-erupcionado.

\section{INTRODUÇÃO}

De acordo com os dados da Organização Mundial de Saúde, a maloclusão, juntamente com a cárie, doença periodontal e o câncer de boca, tem constituido um dos problemas de saúde oral mais prevalentes na população ${ }^{26}$. A prevenção destes distúrbios é o objetivo da Odontologia na busca pela promoção de saúde bucal.

Grande número de casos de maloclusão tem sua origem durante o período da dentadura mista, que se estende dos 6 aos 12 anos de idade. Nesta fase, não há previsão de crescimento ósseo na re- gião mesial aos primeiros molares permanentes, ficando estabelecido o espaço que acomodará os dentes remanescentes que ainda vão irromper ${ }^{3}$.

Métodos diversos têm sido propostos para avaliar o espaço presente no arco dentário e o espaço requerido para acomodação e alinhamento das unidades permanentes que irão irromper ${ }^{7,27}$. As primeiras tentativas de estimar o tamanho dos dentes foram fundamentadas em tabelas de cálculos propostas por Black em $1902^{8}$. Outras técnicas utilizaram a medição direta das unidades dentárias em radiografias ${ }^{19,21,22,32}$, o uso de tabelas ou equações de predição ${ }^{1,6,11,17,20,31}$ e a associação de

$\dagger$ Trabalho realizado no Programa Especial de Treinamento - PET - da Faculdade de Odontologia da Universidade Federal da Bahia.

* Cirurgiãs-Dentistas.

** Professora Adjunta Doutora do Departamento de Odontologia Social; *** Professora Adjunta Doutora do Departamento de Diagnóstico e Terapêutica - Faculdade de Odontologia da Universidade Federal da Bahia. 
MARChIONNI, V. M. T.; SILVA, M. C. de A.; ARAÚJO, T. M. de; REIS, S. R. de A. Avaliação da efetividade do método de TanakaJohnston para predição do diâmetro mésio-distal de caninos e pré-molares não-irrompidos. Pesqui Odontol Bras, v. 15, n. 1, p. 35-40, jan./mar. 2001 .

medidas realizadas em radiografias e em unidades já irrompidas ${ }^{1,10,16,18,30}$.

O método específico de predição proposto por TANAKA; JOHNSTON ${ }^{31}$ (1974) preconiza a utilização de metade da somatória do diâmetro mésio-distal dos incisivos inferiores permanentes, acrescido de um valor predeterminado, que corresponde a $10,5 \mathrm{~mm}$ para um hemi-arco inferior, e $11,0 \mathrm{~mm}$ para um hemi-arco superior.

Tendo como propósito avaliar o método preconizado por Tanaka-Johnston para a predição do diâmetro mésio-distal de caninos e pré-molares não-irrompidos, os autores deste trabalho objetivaram: verificar a efetividade do referido método; estabelecer e comparar a precisão desta técnica quando aplicada a indivíduos dos sexos masculino e feminino; analisar a efetividade das fórmulas de Tanaka-Johnston nos diferentes grupos raciais na cidade de Salvador - BA e observar a exatidão do método comparando-se os lados direito e esquerdo dos arcos dentários.

\section{MATERIAL E MÉTODO}

Para realização desta pesquisa, foi selecionada uma amostra constituída de 98 indivíduos de diferentes raças, sendo 53 do sexo feminino e 45 do sexo masculino, com idade variando entre 13 a 29 anos. Todos deveriam apresentar os dentes permanentes completamente irrompidos até segundos molares, ausência de perda aparente de substância dentária por desgastes, cáries ou restaurações proximais e ausências de alterações dentárias de forma e tamanho, pois assim permitir-se-ia a mensuração no diâmetro mésio-distal real da coroa da unidade dentária. Além disso, não deveriam ter feito uso de aparelho ortodôntico, por existirem algumas técnicas que preconizam o desgaste proximal das unidades dentárias, o que comprometeria o tamanho real da coroa das mesmas.

A classificação das raças, segundo AZEVÊDO ${ }^{5}$ (1980), pela qual os individuos são distribuídos em raça branca, mulato claro, mulato médio, mulato escuro e negra é feita de acordo com a pigmentação da pele, cor e tipo de cabelo e conformação de lábios e nariz.

Auferiu-se o diâmetro mésio-distal dos incisivos permanentes inferiores, caninos e pré-molares superiores e inferiores diretamente na cavidade oral dos indivíduos, com o auxílio de um paquímetro digital de marca Mitutoyo, modelo CD.6"P, Digimatic, $\mathrm{n}^{\circ}$ 500-351. O paquímetro era posicionado o mais perpendicular possível em relação à coroa clínica da unidade dentária, com suas pontas ativas tocando nas faces proximais (mesial e distal) de forma que abrangesse o seu maior diâmetro mésio-distal. Quando a posição da unidade não permitia o ajuste do paquímetro desta forma, a medição era feita com o instrumento paralelo à coroa clínica da unidade no sentido ocluso-gengival.

Todas as medições foram realizadas por um examinador, o qual foi submetido a uma calibração prévia no sentido de evitar discrepâncias. As medidas individuais foram catalogadas em fichas previamente elaboradas e personalizadas.

Ao somatório do diâmetro mésio-distal dos incisivos inferiores permanentes, aplicou-se a fórmula de Tanaka-Johnston calculando-se o valor da predição do diâmetro mésio-distal de caninos e prémolares não-irrompidos.

Foi feita a análise estatística dos dados para obtenção do coeficiente de correlação entre os valores reais e os estimados pelo método de Tanaka-Johnston para avaliar a efetividade do mesmo. Considerou-se cada arco dentário de forma distinta, já que o método de Tanaka-Johnston utiliza uma fórmula para o arco dentário superior e outra para o arco dentário inferior. Para os estudos estatísticos, mediu-se o grau de correlação (r) entre as variáveis (mensuradas ao mínimo em escala ordinal) de interesse através do teste de Pearson ou de Spearman (p), conforme os pressupostos exigidos.

\section{RESULTADOS}

Para a análise dos resultados, comparou-se o valor real de caninos e pré-molares permanentes não-irrompidos com os valores obtidos pelo método de Tanaka-Johnston, avaliando, separadamente, os sexos, as raças e os lados direito e esquerdo em ambos os arcos dentários.

A Tabela 1 demonstra que o coeficiente de correlação revelou-se maior para o arco dentário inferior $(0,60)$ que para o arco dentário superior $(0,50)$.

Os Gráficos 1 e 2 ilustram a dispersão que me-

TABELA 1 - Coeficiente de correlação entre os valores reais e os previstos pelo método de Tanaka-Johnston para o somatório do diâmetro mésio-distal de caninos e pré-molares, na amostra de 98 indivíduos.

\begin{tabular}{c|c|c}
\hline \hline \multirow{2}{*}{ Medidas } & \multicolumn{2}{|c}{ Real versus Tanaka-Johnston } \\
\cline { 2 - 3 } & Superior & Inferior \\
\hline $\mathrm{n}(\Sigma \mathrm{C}, \mathrm{PMs})^{*}$ & 196 & 196 \\
\hline $\mathrm{r}$ & 0,50 & 0,60 \\
\hline Valor de $\mathrm{p}$ & 0,0001 & 0,0001 \\
\hline \hline
\end{tabular}

* Somatório do diâmetro mésio-distal de caninos e prémolares. 
MARChIONNI, V. M. T.; SILVA, M. C. de A.; ARAÚJO, T. M. de; REIS, S. R. de A. Avaliação da efetividade do método de TanakaJohnston para predição do diâmetro mésio-distal de caninos e pré-molares não-irrompidos. Pesqui Odontol Bras, v. 15, n. 1, p. 35-40, jan./mar. 2001.

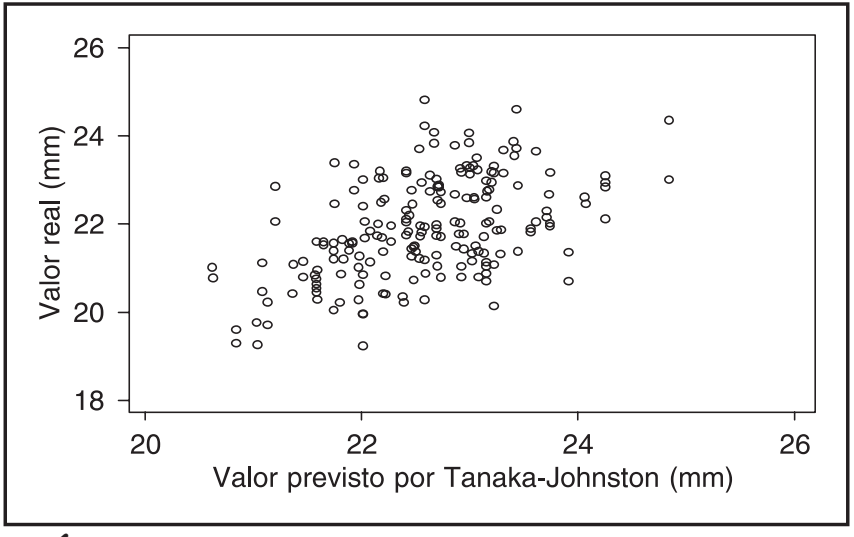

GRÁFICO 1 - Diagrama de dispersão dos valores reais e dos previstos pelo método de Tanaka-Johnston para o diâmetro mésio-distal de caninos e pré-molares para o arco superior.

lhor caracteriza os coeficientes de correlação para os arcos dentários superior e inferior, respectivamente. Comparando-se a distribuição dos pontos entre estes gráficos, observa-se que, à medida que o valor real aumenta, o valor previsto por Tanaka-Johnston também aumenta; no entanto, o diagrama do Gráfico 2 revela uma distribuição mais linear, determinando um maior coeficiente de correlação para o arco dentário inferior.

De acordo com os resultados da Tabela 2, os maiores coeficientes de correlação foram para o arco dentário inferior tanto para o sexo masculino $(0,59)$ quanto para o sexo feminino $(0,60)$, enquanto o menor coeficiente de correlação foi para o arco dentário superior do sexo masculino $(0,46)$.

A Tabela 3 expressa os coeficientes de correlação obtidos para cada raça. Observa-se que, para o arco dentário superior, o maior coeficiente de correlação foi para a raça mulato escuro $(0,67)$ e o menor para a raça branca $(0,44)$. Para o arco dentário inferior, o maior coeficiente de correlação foi para a raça mulato claro $(0,74)$ e a menor foi para a raça mulato médio $(0,45)$. Pode-se observar que o maior coeficiente de correlação obtido foi para a raça mulato claro $(0,74$, no arco dentário inferior) e o menor foi para a raça branca $(0,44$, no arco dentário superior).

Em relação aos coeficientes de correlação dos valores reais e os previstos por Tanaka-Johnston para o diâmetro mésio-distal de caninos e pré-molares dos lados direito e esquerdo (Tabela 4), observa-se que o maior coeficiente de correlação foi para o lado esquerdo do arco dentário inferior $(0,61)$ e o menor para o lado esquerdo do arco dentário superior $(0,48)$.

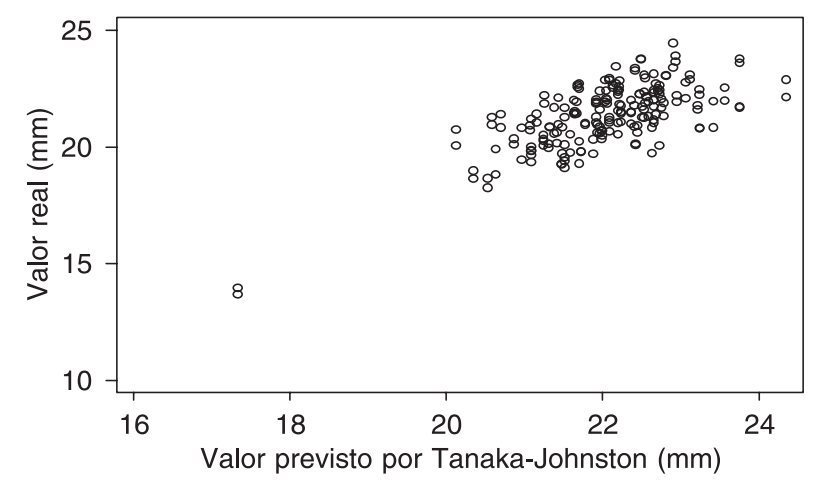

GRÁFICO 2 - Diagrama de dispersão dos valores reais e dos previstos pelo método de Tanaka-Johnston para o diâmetro mésio-distal de caninos e pré-molares para o arco inferior.

TABELA 2 - Distribuição do coeficiente de correlação para o somatório do diâmetro mésio-distal de caninos e pré-molares entre os valores reais e previstos pelo método de Tanaka-Johnston para 45 indivíduos do sexo masculino e 53 do sexo feminino.

\begin{tabular}{c|l|l|l|l}
\hline \hline \multirow{2}{*}{ Medidas } & \multicolumn{2}{|c|}{ Masculino } & \multicolumn{2}{c}{ Feminino } \\
\cline { 2 - 5 } & Superior & Inferior & Superior & Inferior \\
\hline $\mathrm{n}\left(\sum \mathrm{C}, \mathrm{PMs}\right)$ & 90 & 90 & 106 & 106 \\
\hline $\mathrm{r}$ & 0,46 & 0,59 & 0,52 & 0,60 \\
\hline Valor de $\mathrm{p}$ & 0,0001 & 0,0001 & 0,0001 & 0,0001 \\
\hline \hline
\end{tabular}

\section{DISCUSSÃO}

A avaliação do espaço presente no arco dentário e do espaço requerido para acomodação das unidades não-irrompidas é uma prática da Ortodontia Preventiva, quando se analisa o paciente na fase da dentadura mista ${ }^{12,23}$.

No que diz respeito à idade, os trabalhos que limitaram a faixa etária da amostra mostraram-se eficientes, uma vez que tal controle é necessário para impedir alguma discrepância nas mensurações baseadas na perda de substância dentária pelo desgaste fisiológico ${ }^{2,15}$. Nesta pesquisa, a faixa etária dos indivíduos estudados variou de 13 a 29 anos de idade.

Assim como nos estudos desenvolvidos por AL-KHADRA $^{2}$ (1993), DALIJAN et al. ${ }^{14}$ (1995) e PARDO et al. ${ }^{24}$ (1998), foram excluídos desta amostra os indivíduos que usaram ou estavam fazendo uso de aparelho ortodôntico. CROSBY; ALEXAN$\operatorname{DER}^{13}$ (1989), entretanto, utilizaram em seus estudos pacientes em tratamento ortodôntico com au- 
MARChIONNI, V. M. T.; SILVA, M. C. de A.; ARAÚJO, T. M. de; REIS, S. R. de A. Avaliação da efetividade do método de TanakaJohnston para predição do diâmetro mésio-distal de caninos e pré-molares não-irrompidos. Pesqui Odontol Bras, v. 15, n. 1, p. 35-40, jan./mar. 2001 .

TABELA 3 - Distribuição do coeficiente de correlação para o somatório do diâmetro mésio-distal de caninos e pré-molares entre os valores reais e previstos pelo método de Tanaka-Johnston para indivíduos da raça branca, mulato claro, mulato médio, mulato escuro e negra, na amostra estudada.

\begin{tabular}{c|l|l|l|l|l|l|l|l|l|l}
\hline \hline \multirow{2}{*}{ Medidas } & \multicolumn{2}{|c|}{ Branca } & \multicolumn{2}{c|}{ Mulato claro } & \multicolumn{2}{c|}{ Mulato médio } & \multicolumn{2}{c|}{ Mulato escuro } & \multicolumn{2}{c}{ Negra } \\
\cline { 2 - 12 } & Superior & Inferior & Superior & Inferior & Superior & Inferior & Superior & Inferior & Superior & Inferior \\
\hline $\mathrm{n}\left(\sum \mathrm{C}, \mathrm{PMs}\right)$ & 38 & 38 & 44 & 44 & 42 & 42 & 36 & 36 & 36 & 36 \\
\hline $\mathrm{r}$ & 0,44 & 0,57 & 0,56 & 0,74 & 0,48 & 0,45 & 0,67 & 0,71 & 0,51 & 0,55 \\
\hline Valor de $\mathrm{p}$ & 0,0062 & 0,0002 & 0,0001 & 0,0001 & 0,0013 & 0,0025 & 0,0001 & 0,0001 & 0,0014 & 0,0005 \\
\hline \hline
\end{tabular}

TABELA 4 - Distribuição do coeficiente de correlação para o somatório do diâmetro mésio-distal de caninos e pré-molares entre os valores reais e previstos pelo método de Tanaka-Johnston para os lados direito e esquerdo de 98 indivíduos.

\begin{tabular}{c|l|l|l|l}
\hline \hline \multirow{2}{*}{ Medidas } & \multicolumn{2}{|c|}{ Lado direito } & \multicolumn{2}{c}{ Lado esquerdo } \\
\cline { 2 - 5 } & Superior & Inferior & Superior & Inferior \\
\hline $\mathrm{n}\left(\sum \mathrm{C}, \mathrm{PMs}\right)$ & 98 & 98 & 98 & 98 \\
\hline $\mathrm{r}$ & 0,52 & 0,60 & 0,48 & 0,61 \\
\hline Valor de $\mathrm{p}$ & 0,0001 & 0,0001 & 0,0001 & 0,0001 \\
\hline \hline
\end{tabular}

sência de desgaste interproximal ou extração dentária, visto que as medições foram realizadas em ambulatório com controle dos procedimentos ortodônticos.

A inclusão de variáveis como raça, sexo e idade em alguns estudos oferece maior segurança para avaliar a efetividade de uma técnica de predição, quando aplicada a diferentes populações ${ }^{9,15}$. TANAKA; JOHNSTON ${ }^{31}$ (1974) questionaram a validade da pesquisa realizada por MOYERS ${ }^{20}$ (1958) pela caracterização insuficiente da amostra, já que a única especificação fornecida foi a respeito da descendência dos indivíduos que a compunham.

Baseando-se na premissa de que a raça é um fator que contribui para a variabilidade do diâmetro mésio-distal de unidades dentárias permanentes, e considerando que as fórmulas de Tanaka-Johnston foram elaboradas a partir de uma amostra de indivíduos de descendência européia, alguns autores propuseram-se a verificar a efetividade do referido método em diferentes grupos étnicos, como o trabalho de INGERVALL; LENNARTSSON $^{17}$ (1978) em suecos, de FRANKEL; BENZ ${ }^{15}$ (1986) em americanos negros, a pesquisa de AL-KHADRA $^{2}$ (1993) na população da Arábia Saudita e a de PARRA et al. ${ }^{25}$ (1998) em indivíduos colombianos.
Ao avaliar a efetividade do método de predição preconizado por TANAKA; JOHNSTON ${ }^{31}$ (1974) nas diferentes raças da cidade de Salvador - Bahia, constatamos que este foi eficiente para todas as raças analisadas, na amostra estudada. Nesta pesquisa, a raça branca, no arco dentário superior, obteve o menor coeficiente de correlação $(0,44)$, e no arco dentário inferior este coeficiente foi menor $(0,57)$ que das raças mulato claro $(0,74)$ e mulato escuro $(0,71)$.

Segundo AL-KHADRA² (1993), a diferença de resultados entre diferentes populações é decorrente das origens étnicas das amostras, considerando-se a possibilidade de haver uma variação morfológica no tamanho dos dentes. Neste trabalho, foi observado que os indivíduos da raça negra apresentaram dentes maiores que das demais raças. Na avaliação da efetividade da equação proposta por TANAKA; JOHNSTON ${ }^{31}$ (1974) para predição do diâmetro mésio-distal de caninos e pré-molares não irrompidos, em indivíduos negros, FRANKEL; BENZ ${ }^{15}$ (1986) constataram coeficientes de correlação maiores $(0,65$ para o arco maxilar e 0,70 para o arco mandibular) que os encontrados nesta pesquisa.

Em relação ao sexo, nossos achados estão de acordo com alguns autores que encontraram o diâmetro mésio-distal das unidades permanentes maiores em indivíduos do sexo masculino que do sexo feminino $0^{4,19,23,28,33}$.

Não obstante, outra questão fundamental a ser discutida é a comparação das médias do somatório do diâmetro mésio-distal de caninos e pré-molares do lado esquerdo com o lado direito em um mesmo arco dentário, o que confere um suporte científico mais apurado e específico no estudo da predição do tamanho de unidades dentárias. TANAKA; JOHNSTON $^{31}$ (1974), STALEY et al. ${ }^{29}$ (1983) e PAULA et al. $^{23}$ (1995) não consideraram os lados separadamente, sugerindo uma simetria bilateral. Os achados desta pesquisa discordaram desta 
MARChIONNI, V. M. T.; SILVA, M. C. de A.; ARAÚJO, T. M. de; REIS, S. R. de A. Avaliação da efetividade do método de TanakaJohnston para predição do diâmetro mésio-distal de caninos e pré-molares não-irrompidos. Pesqui Odontol Bras, v. 15, n. 1, p. 35-40, jan./mar. 2001.

afirmativa, uma vez que os coeficientes de correlação para o lado esquerdo diferiram daqueles para o lado direito. Constatou-se que o arco dentário superior do lado esquerdo apresentou menor coeficiente de correlação $(0,48)$, retratando que, na amostra estudada, o método foi mais efetivo para o lado direito do arco dentário superior.

Trabalhar com probabilidades que exijam exatidão torna-se dificil, pois a anatomia humana é dotada de variações. Diversos são os métodos que estimam o valor do diâmetro mésio-distal de caninos e pré-molares por meio de tabelas, equações e radiografias. Obter, através destes, o valor mais próximo do real é um desafio, pois todos correm o risco de falhar. A avaliação da efetividade dos mesmos não é um exame para aprová-los ou reprová-los, mas um mecanismo para verificar como esses métodos são responsáveis por um diagnóstico seguro. Assim sendo, aliado aos métodos de predição, deve existir o bom senso do profissional, a fim de que o diagnóstico seja elaborado de forma eficaz.

\section{CONCLUSÕES}

Com base na metodologia utilizada e nos resul- tados obtidos na amostra estudada, concluiu-se que:

1. a diferença entre os coeficientes de correlação do arco dentário superior e inferior foi pequena, comprovando a efetividade do método de Tanaka-Johnston para a predição do diâmetro mésio-distal de caninos e pré-molares não-irrompidos;

2. mesmo não existindo fórmulas distintas para o sexo masculino e o feminino, e ter sido obtida uma leve diferença entre os coeficientes de correlação, o método de Tanaka-Johnston apresentou-se efetivo para ambos;

3. apesar de o método de Tanaka-Johnston ter sido desenvolvido a partir de uma amostra de descendentes de ancestrais europeus, o maior coeficiente de correlação obtido foi para a raça mulato claro $(0,74)$, seguida da raça mulato escuro $(0,71)$, sugerindo que este método pode ser utilizado para diferentes raças;

4. não foi obtida uma simetria entre os lados direito e esquerdo como proposto por Tanaka-Johnston; entretanto, a assimetria encontrada não comprometeu a efetividade do método para ambos os lados dos arcos dentários.

MARCHIONNI, V. M. T.; SILVA, M. C. de A.; ARAÚJO, T. M. de; REIS, S. R. de A. Evaluation of the effectiveness of the Tanaka-Johnston method for prediction of the mesiodistal diameter of unerupted canines and premolars. Pesqui Odontol Bras, v. 15, n. 1, p. 35-40, jan./mar. 2001.

\begin{abstract}
In the present study, the authors evaluated the Tanaka-Johnston method of prediction, with the objective of verifying its effectiveness for the right and left sides, for both genders and for the white, light mulatto, medium mulatto, dark mulatto and black races in the city of Salvador, Bahia. The mesiodistal diameter of the inferior permanent incisors, canines and premolars that had already erupted was measured with the aid of a digital caliper rule in the oral cavity of 98 individuals (45 males and 53 females), aging from 13 to 29 years. The results obtained with the application of the Tanaka-Johnston formulas were compared with the real values through statistical tests. After the analysis of the results, it was observed that the correlation between the estimated values and the real values was greater for females than it was for males. As to race, the coefficients of correlation were satisfactory. They were greater in the superior arch for the dark mulatto race (0.67), and in the inferior arch for the light mulatto race (0.74). Considering the sides, the findings revealed a greater coefficient of correlation for the inferior arch on the left side (0.61). It was concluded that the Tanaka-Johnston method, in spite of originating from a sample of European descendants, is indicated for the prediction of the mesiodistal diameter of canines and premolars in the different races evaluated in this research. The effectiveness of the referred method was also checked for both genders and sides in the studied sample.
\end{abstract}

UNITERMS: Dentition, mixed; Orthodontics, interceptive; Tooth, unerupted.

\section{REFERÊNCIAS BIBLIOGRÁFICAS}

1. ABE, K.; SUZUKI, A.; TAKAHAMA, Y. Prediction of the coronal size of third molars by factor and multiple regression analyses. Am J Orthod Dentofacial Orthop, v. 109, n. 1, p. 79-85, Jan. 1996.

2. AL-KHADRA, B. H. Prediction of the size of unerupted canines and premolars in a Saudi Arab population. Am J
Orthod Dentofacial Orthop, v. 104, n. 4, p. 369-372, Oct. 1993.

3. ARAÚJO, M. Ortodontia para clínicos. 3. ed., São Paulo : Santos, 1986. p. 129-148.

4. ARYA, B. S.; SAVARA, B. S.; THOMAS, D.; CLARKSON, Q. Relation of sex and occlusion to mesiodistal tooth size.

Am J Orthod, v. 66, n. 5, p. 479-486, Nov. 1974.

5. AZEVÊDO, E. S. Subgroup studies of black admixture 
MARChIONNI, V. M. T.; SILVA, M. C. de A.; ARAÚJO, T. M. de; REIS, S. R. de A. Avaliação da efetividade do método de TanakaJohnston para predição do diâmetro mésio-distal de caninos e pré-molares não-irrompidos. Pesqui Odontol Bras, v. 15, n. 1, p. 35-40, jan./mar. 2001 .

within a mixed population of Bahia, Brazil. Ann Hum Genet, v. 44, n. 55, p. 55-60, July 1980.

6. BALLARD, M. L.; WYLIE, W. L. Mixed dentition case analysis - estimating size of unerupted permanent teeth. Am J Orthod, v. 33, p. 754-759, 1947.

7. BATTAGEL, J. M. The assessment of crowding without the need to record arch perimeter. Part I: arches with acceptable alignment. $\mathbf{B r} \mathbf{J}$ Orthod, v. 23, n. 2, p. 137-144, May 1996.

8. BERTHOLD, T. B.; CLOSS, L. Q.; NYS, P. P.; CAMINHA, J. A. Tanaka-Johnston - um método simplificado e efetivo para a predição de tamanho de dentes permanentes não erupcionados. Rev Fac Odontol Porto Alegre, v. 34, n. 1, p. 28-31, set. 1993.

9. BISHARA, S. E.; JAKOBSEN, J. R.; ABDALLAH, E. M.; GARCIA, A. F. Comparisons of mesiodistal and buccolingual crown dimensions of the permanent teeth in three populations from Egypt, Mexico and the United States. Am J Orthod Dentofacial Orthop, v. 96, n. 5, p. 416-422, Nov. 1989.

10. BISHARA, S. E.; STALEY, R. N. Mixed-dentition mandibular arch length analysis: a step-by-step approach using the revised Hixon-Oldfather prediction method. Am $\mathbf{J}$ Orthod, v. 86, n. 2, p. 130-135, Aug. 1984.

11. CAREY, C. W. Linear arch dimension and tooth size. Am J Orthod, v. 35, p. 762-775, Oct. 1949.

12. CLOSS, L. Q.; NYS, P. P.; CAMINHA, J. A. N.; BERTHOLD, T. B. Avaliação dos métodos de predição do diâmetro de caninos e pré-molares não erupcionados de Moyers e o de Tanaka/Johnston. Ortodont Paranaen, v. 13, n. 1, p. 1-6, jan./jun. 1992.

13. CROSBY, D. R.; ALEXANDER, C. G. The occurrence of tooth size discrepancies among different malocclusion groups. Am J Orthod Dentofacial Orthop, v. 95, n. 6, p. 457-461, June 1989.

14. DALIDJAN, M.; SAMPSON, W.; TOWNSEND, G. Prediction of dental arch development: an assessment of point's index in three human populations. Am J Orthod Dentofacial Orthop, v. 107, n. 5, p. 465-475, May 1995.

15. FRANKEL, H. H.; BENZ, E. M. Mixed dentition analysis for black Americans. Pediatr Dent, v. 8, n. 3, p. 226-230, Sept. 1986.

16. HIXON, E. H.; OLDFATHER, R. E. Estimation of the sizes of unerupted cuspid and bicuspid teeth. Angle Orthod, v. 28 , n. 4 , p. 236-241, Oct. 1958.

17. INGERVALL, B.; LENNARTSSON, B. Prediction of breadth of permanent canines and premolars in the mixed dentition. Angle Orthod, v. 48, n. 1, p. 62-69, Jan. 1978.

18. KAPLAN, R. G.; SMITH, C. C.; KANAREK, P. H. An analysis of three mixed dentition analyses. J Dent Res, v. 56, n. 11 , p. 1337-1343, Nov. 1977.

19. MOORREES, C. F. A.; THOMSEN, S. O.; JENSEN, E.; YEN, P. K. Mesiodistal crown diameters of the deciduos and permanent teeth in individuals. J Dent Res, v. 36, n. 1, p. 39-46, Feb. 1957.

20. MOYERS, R. E. Ortodontia. 4. ed., Rio de Janeiro : Guanabara Koogan, 1991. p. 187-207.

21. NANCE, H. N. The limitations of orthodontic treatment - I. Mixed dentition diagnosis and treatment. Am J Orthod Oral Surg, v. 33, n. 4, p. 177-223, Apr. 1947.

22. OliveIRA, A. G.; PINZAN, A.; HENRIQUES, J. F. C. Avaliação da análise de Moyers para predição do tamanho mésio-distal dos caninos e pré-molares, não-irrompidos, na dentadura mista, em pacientes da região de Bauru. Ortodontia, v. 24, n. 1, p. 18-23, jan./abr. 1991.

23. PAUlA, S.; ALMEIDA, M. A. O.; LEE, P. C. F. Prediction of mesiodistal diameter of unerupted lower canines and premolars using $45^{\circ}$ cephalometric radiography. Am J Orthod Dentofacial Orthop, v. 107, n. 3, p. 309-314, Mar. 1995.

24. PARDO, M. A.; PARRA, M. I.; YEZIORO, S. Aplicación de cinco análisis de dentición mixta en una muestra de niños colombianos. In: PRIMER ENCUENTRO LATINOAMERICANO DE INVESTIGACIÓN EN ORTODONCIA, Catagena. v. 1, n. 1, p. 40-7, 1998.

25. PARRA, M. I.; PARDO, M. A.; YEZIORO, S. Predicción del tamaño mesiodistal de los caninos permanentes y premolares no erupcionados en una muestra de niños colombianos. In: PRIMER ENCUENTRO LATINOAMERICANO DE INVESTIGACIÓN EN ORTODONCIA, Catagena. v. 1, n. 1, p. 48-57, 1998.

26. PINTO, V. G. Saúde bucal: Odontologia Social e Preventiva. 3. ed. Planejamento São Paulo: Santos, 1992. p. 21-35.

27. RABERIN, M.; LAUMON, B.; MARTIN, J.; BRUNNER, F. Dimensions and form of dental arches in subjects with normal occlusions. Am J Orthod Dentofacial Orthop, v. 104, n. 1, p. 67-72, July 1993.

28. RICHARDSON, E. R.; MALHOTRA, S. K. Mesiodistal crown dimension of the permanent dentition of American Negroes. Am J Orthod, v. 68, n. 2, p. 157-164, Aug. 1975.

29. STALEY, R. N.; HU, P.; HOAG, J. F.; SHELLY, T. H. Prediction of the combined right and left canine and premolar widths in both arches of the mixed dentition. Pediatr Dent, v. 5, n. 1, p. 57-60, 1983.

30. STALEY, R. N.; KERBER, P. E. A revision of the Hixon and Oldfather mixed-dentition prediction method. Am $\mathbf{J}$ Orthod, v. 78, n. 3, p. 296-302, Sept. 1980.

31. TANAKA, M. M.; JOHNSTON, L. E. The prediction of the size of unerupted canines and premolares in a contemporary orthodontic population. J Am Dent Assoc, v. 88, n. 4, p. 798-801, Apr. 1974.

32. WATSON, D. H. A rapid and accurate assessment of the mesiodistal dimensions of any unerupted permanent tooth. Int J Orthod, v. 10, n. 3, p. 91-92, Sept. 1972.

33. YUEN, K. K.; TANG, E. L.; SO, L. L. Mixed dentition analysis for Hong Kong Chinese. Angle Orthod, v. 68, n. 1, p. 21-28, Sept. 1998.

Recebido para publicação em 02/02/00 Enviado para reformulação em 20/07/00 Aceito para publicação em 17/11/00 